\title{
Análise das Situações Simuladas da Prática Médica
}

\author{
Review of Medical Practice Simulations
}

\author{
Roseli Ferreira da Silval \\ Aline Guerra Aquilante \\ Sílvia Helena Zem-Mascarenhas \\ Renata Giannecchini Bongiovanni Kishi \\ Cássia Regina Rodrigues Vargal
}

\section{PALAVRAS-CHAVE: \\ - Currículo. \\ - Educação Médica. \\ - Avaliação. \\ - Aprendizagem Baseada em Problemas.}

\section{KEYWORDS:}

- Curriculum.

- Education, Medical.

- Evaluation.

- Problem-Based Learning.

Recebido em:16/05/2011

Reencaminhado em: 09/09/2011

Aprovado em: 04/11/2011

REVISTA BRASILEIRA DE EDUCAÇÃO MÉDICA

\section{RESUMO}

Considerando a importância das atividades curriculares para a formação médica, este estudo teve por objetivo identificar e refletir sobre a construção das situações utilizadas na atividade curricular Estações de Simulação (ES) pela primeira turma do curso médico da Universidade Federal de São Carlos/ SP-UFSCar, de 2006 a 2009. Trata-se de um estudo de caso do tipo descritivo, que analisa 57 situações de ES e suas ementas. Os resultados revelaram predomínio de estações na área de Saúde do Adulto e Idoso, e Saúde da Família e Comunidade. Foram analisadas outras variáveis, como idade, gênero, fases do processo saúde-doença, cenário de atenção à saúde e áreas do conhecimento, entre outras. Foi possível identificar a necessidade de readequar as ES do curso médico da UFSCar, elaboradas de 2006 a 2009. Este estudo contribuiu com a formulação de variáveis e critérios que devem orientar a construção das ES, para que estas representem o melhor possível a realidade da prática profissional.

\footnotetext{
Considering the importance of curricular activities for medical training, this study aimed to identify and reflect on the construction of the situations used in activities at the Simulation Stations by the first class of the São Carlos Federal University medical school (SP-UFSCar), from 2006 to 2009. This is a descriptive case study, where 57 simulations and their programs were analyzed. The results showed a predominance of stations in the area of Adult and Elderly Health and Community and Family Health. Other variables were analyzed such as age, gender, stages of illness and health, the health care setting, areas of knowledge and others. The need was identified to restructure the SS of the UFSCar Medical School, developed between 2006 and 2009. This study has contributed to formulating some criteria and variables that should guide the construction of the SS, so that they can best represent the reality of medical practice.
} 


\section{INTRODUÇÃO}

Desde as décadas de 1970 e 1980, tem se dado muita atenção ao desenvolvimento e avaliação de competências profissionais em diversas especialidades médicas ${ }^{1}$. Todavia, somente após a introdução de pacientes simulados, há 40 anos, foi possível avaliar o desempenho dos profissionais médicos com base em critérios objetivos, estruturados e padronizados ${ }^{2}$. Essa introdução potencializou o ensino por meio de simulações na educação médica, pois os pacientes simulados constituem uma estratégia pedagógica que auxilia também no desenvolvimento da comunicação e do procedimento clínico, e ainda permite ao estudante a análise de seu desempenho ${ }^{3,4}$.

A incorporação das simulações aos cenários de ensino-aprendizagem possibilitou que as escolas criassem diversos modelos, e várias nuances dessa ferramenta estão sendo desenvolvidas e utilizadas nos diferentes contextos do ensino médico ${ }^{5-7}$

Embora estruturada em cada instituição educacional de diferentes formas, a Simulação tem se estabelecido, tanto na graduação como na pós-graduação lato sensu, como um instrumento que assegura certo grau de autonomia ao estudante, graças a um ambiente controlado e seguro. Também promove oportunidades práticas que favorecem a aquisição de habilidades psicomotoras e psicológicas. Além disso, essa ferramenta permite a combinação deliberada de práticas gerais e específicas com a oportunidade de feedback e promove generalizações efetivas das situações simuladas no processo ensino-aprendizagem dos cursos para os cenários reais, despertando no aluno o senso de autoavaliação e autorreflexão sobre sua eficácia ${ }^{8,9}$.

Nas esferas nacionais e internacionais, a adesão a esta estratégia se sustenta pelo pressuposto de que o conhecimento se constrói a partir dos significados e significantes das relações socioculturais e da própria ação humana ${ }^{10-13}$. Além disso, o uso de simulações possibilita a construção de novos saberes relativos aos processos de cuidado das pessoas a partir da própria vivência, proporcionando aos estudantes o desenvolvimento de capacidades cognitivas, psicomotoras e atitudinais para a identificação de necessidades de saúde e a elaboração de planos de cuidados individuais ${ }^{14}$.

Por esta razão, na atividade de Estação de Simulação da Prática Profissional (ESPP), desenvolvida na Universidade Federal de São Carlos (UFSCar), o conhecimento é construído a partir de situações simuladas relevantes na realidade da prática médica, organizadas para serem desenvolvidas no ambiente protegido e estruturado das simulações. Nessas atividades, o professor, no papel de facilitador da aprendizagem, avalia o desempenho dos estudantes, possibilitando a estes a oportunidade de desenvolver habilidades clínicas e de comunicação, o manejo de recursos e o relacionamento interpessoal médico-paciente. Permite, também, desenvolver destrezas para resolver problemas, analisar e sintetizar as informações obtidas antes do emprego destas com pacientes reais ${ }^{15,16}$

Todavia, para que haja efetividade na utilização desse recurso pedagógico, é necessário um planejamento cuidadoso, recursos financeiros e capacitação periódica dos professores e pacientes simulados/atores ${ }^{4}$

\section{O PLANEJAMENTO DAS ESPP NA UFSCAR}

No planejamento das Estações de Simulação (ES), os professores selecionam os conteúdos - cognitivos, psicomotores, atitudinais - com base no que está previsto para cada ciclo do currículo no projeto pedagógico. Estes conteúdos são ordenados numa matriz de intencionalidade que orienta a construção da situação a ser trabalhada em cada uma das ES da série. Assim, a matriz contém os objetivos e o foco da aprendizagem em cada uma das áreas de competência estabelecidas para o curso.

A partir desse delineamento é elaborado o caso da estação simulada, redigido de forma clara, para cada uma das situações, contendo o contexto, a ementa, o papel a ser interpretado pelo paciente/ator e a tarefa do estudante. Cada caso apresenta o maior número possível de informações sobre a história da família e da vida da pessoa.

O contexto de cuidado em saúde da Estação de Simulação (ES) deve coincidir com o próprio cenário no qual o estudante está inserido para o desenvolvimento das atividades da Unidade Educacional da Prática Profissional (UEPP). Nos dois primeiros anos do curso, ou seja, no primeiro ciclo, os cenários de aprendizagem da UEPP são as Unidades de Saúde da Família (USF). No segundo ciclo (terceira e quarta séries), além das USF, os estudantes desenvolvem atividades nas Unidades Básicas de Saúde (UBS), nas áreas de saúde da mulher, criança e adulto. Assim, a atividade curricular de Estações de Simulação segue a mesma lógica e ordem dos cenários da UEPP, preparando os estudantes para exercerem as atividades na prática, em contexto real.

\section{O PROCESSO PEDAGÓGICO DAS ESPP NA UFSCAR}

As atividades das ESPP são divididas em dois momentos: simulação e acompanhamento. No primeiro, um estudante realiza história e exame físico e elabora plano de cuidados, sendo observado pelo professor e por outro estudante. Após a simulação, o paciente/ator emite seus sentimentos acerca da atuação do estudante e deixa o ambiente. Em seguida, o estudante inicia o processo de autoavaliação, expressando suas considerações em relação ao seu desempenho. Nesse instante, 
o estudante apresenta uma síntese da situação e as necessidades de saúde que identificou.

A partir disso, o professor explora as fundamentações implícitas e/ou explícitas nas ações executadas, instigando o aluno a analisar o que desenvolveu e o que poderia ter desenvolvido nas dimensões cognitiva, atitudinal e psicomotora. Nessa reflexão, o estudante expõe seu conhecimento prévio e respectivas fundamentações teóricas ${ }^{4}$. Após esses passos, o estudante observador apresenta sua avaliação do colega e depois o professor apresenta seu feedback ao estudante, ou seja, sua avaliação formativa. Vale destacar que o objetivo do professor, durante a exploração da fundamentação, é compreender as dimensões dos conhecimentos prévios que apoiam o desempenho do estudante ${ }^{17}$.

Como produto da simulação, o estudante redige e arquiva em seu portfólio a história clínica, o exame físico, as necessidades de saúde e o plano de cuidados. Esse material é utilizado como disparador no momento do acompanhamento. Neste segundo momento, os estudantes trabalham em pequenos grupos de aprendizagem, com quatro a oito estudantes, e realizam o movimento de síntese provisória e de nova síntese, tal como o pequeno grupo da Aprendizagem Baseada em Problemas (ABP).

Na síntese provisória, o professor, no papel de facilitador, ajuda os estudantes a refletirem sobre as histórias clínicas e desdobramentos das simulações realizadas. Essa reflexão tem como meta a conscientização acerca dos hiatos e fronteiras de conhecimento, resultando primeiro na formulação das hipóteses que fundamentaram a discussão e, posteriormente, na construção de questões de aprendizagem que expressam as necessidades educacionais.

Na nova síntese, os estudantes trazem as sínteses individuais das questões de aprendizagem, fundamentadas na literatura científica. Nessa oportunidade, os estudantes exercitam algumas habilidades psicomotoras, como técnicas de exame físico, tendo o professor, além do papel de facilitador, o de consultor, para redimensionar as deficiências apresentadas.

Todo o processo de aprendizagem de cada estação de simulação, desde a simulação até a finalização do acompanhamento, dura cerca de quatro semanas. O ambiente protegido da ESPP, tanto para o paciente/ator quanto para o estudante, abre espaço para que este aprenda fazendo, errando e aprendendo com os próprios erros. Ao refletir sobre o erro, o aluno pode construir seu aprendizado, por meio da identificação de lacunas em seu conhecimento, e fundamentar cognitivamente suas habilidades ${ }^{4}$.

Esta investigação teve o propósito de evidenciar os princípios e os componentes empregados para formular situações simuladas da prática profissional no contexto da formação médica.

\section{METODOLOGIA}

\section{Campo do Estudo}

O currículo do curso de Medicina da UFSCar é orientado por competência, de modo a promover e acompanhar o desenvolvimento da prática profissional médica. O currículo é integrado, pois propicia a articulação das dimensões teórica e prática, clínica e epidemiológica, das diferentes disciplinas e da aprendizagem com o mundo do trabalho. São utilizadas metodologias ativas, tais como a Aprendizagem Baseada Em Problemas (ABP) e aprendizagem baseada na prática ${ }^{14}$.

As áreas de competência para a atuação médica do egresso do curso de Medicina da UFSCar são: (a) Saúde, com as subáreas: cuidado às necessidades individuais de saúde, e cuidado às necessidades coletivas de saúde; (b) Gestão, com as subáreas: organização do trabalho em saúde e gestão do cuidado; (c) Educação.

O curso está estruturado em três ciclos educacionais. Cada ciclo é organizado por três Unidades Educacionais: Unidade Educacional de Simulação da Prática Profissional, Unidade Educacional de Prática Profissional e Unidade Educacional Eletiva.

Na Unidade Educacional de Prática Profissional (UEPP), o confronto direto com a realidade é o elemento disparador do processo ensino-aprendizagem. Nesse sentido, é desenvolvida em cenários reais e focaliza o desenvolvimento, em contexto, da prática profissional. A Prática Profissional é desenvolvida durante todo o curso, ampliando o domínio e a autonomia na realização dos desempenhos focalizados em cada ano letivo e ciclo.

Na Unidade Educacional Eletiva (UEE), formada por atividades complementares desenvolvidas em períodos preestabelecidos na grade curricular, o estudante - com o apoio de um professor orientador e aprovação do Conselho de Coordenação - elabora seu plano de ensino a partir de suas necessidades educacionais, à luz do perfil de competência, podendo desenvolvê-lo dentro ou fora da universidade.

Na Unidade Educacional de Simulação da Prática Profissional (UESPP), os elementos disparadores do processo ensino-aprendizagem podem ser situações-problema de papel, dramatizações, filmes, situações simuladas da prática profissional e outros recursos que permitam a construção de saberes em cenários protegidos e controlados. Esta Unidade é formada por duas atividades curriculares: Estações de Simulação da Prática Profissional (ESPP) e Situações-Problema (SP).

\section{Objetivo do Estudo}

Identificar e analisar a construção das situações utilizadas na atividade curricular Estações de Simulação pela primeira turma do curso médico da UFSCar, no período de 2006 a 2009. 


\section{Tipo de Estudo}

Pela natureza do objeto de estudo, esta investigação utilizou uma metodologia que se caracteriza como estudo de caso, do tipo descritivo, com abordagens qualitativas e quantitativas. Foram objeto de análise deste estudo as 57 situações destas ES e suas ementas.

\section{Coleta e Análise dos Dados}

Para a realização da coleta de dados foram selecionadas todas as 57 situações trabalhadas com os estudantes da primeira turma, sendo 20 da primeira série, 20 da segunda, 11 da terceira e 6 da quarta série.

Esta investigação faz parte do projeto "A implantação de um currículo de Medicina: uma análise sob a ótica de seus diferentes atores", do grupo de pesquisa "Educação para profissionais de saúde", o qual foi submetido a todos os procedimentos de autorização institucional e recebeu parecer favorável do Comitê de Ética e Pesquisa em Seres Humanos da UFSCar.

\section{Análise e Interpretação dos Dados}

Para a análise dos dados, num primeiro momento foram determinadas as seguintes variáveis de análise: gênero, idade, ocupação (segundo as categorias de ocupação definidas pela Abep Associação Brasileira das Empresas de Pesquisa) ${ }^{18}$; religião e cor de pele segundo classificação do IBGE; desfecho da condição do paciente na situação simulada (alta, seguimento, óbito); fases do processo saúde-doença presentes na situação simulada (promoção, prevenção, tratamento, reabilitação); condição do paciente da situação simulada (saúde ou doença); cenário de atenção à saúde no qual a situação simulada se desenvolvia (atenção primária, secundária, terciária); áreas do conhecimento (segundo disciplinas do curso médico); dimensões das disciplinas presentes na situação simulada (biológica, psicológica, social); e áreas de competência estabelecidas no perfil do curso (cuidado individual, cuidado coletivo, gestão do cuidado, educação).

No que se refere à análise quantitativa, as informações foram analisadas por meio de medidas de frequências absolutas e relativas. A partir desta análise foram feitas algumas considerações qualitativas.

Foram trabalhadas 57 ES de 2006 a 2009 pela Turma I. Houve predomínio de estações na área de conhecimento de Saúde do Adulto e Idoso (35,1\%) e Saúde da Família e Comunidade (33,3\%). Em seguida, apareceram estas duas áreas associadas $(21,1 \%)$, Saúde da Criança $(7,0 \%)$ e Saúde da Mulher (3,5\%). É esperado que a área de Saúde do Adulto e Idoso seja a mais trabalhada, considerando a maior concentração desta faixa etária na população brasileira. A interface entre as diferentes áreas e Saúde da Família e Comunidade é previsível, pois esta área é abrangente e se relaciona com os cuidados de atenção primária às pessoas durante todo o seu ciclo de vida, independentemente do gênero. No entanto, as proporções foram muito desiguais na distribuição por área de conhecimento, e as áreas Saúde Coletiva e Saúde Mental e Educação não foram abordadas nas ES.

As variáveis sociodemográficas dos personagens analisadas foram: gênero, idade, ocupação, religião e cor. Em relação ao gênero, houve equilíbrio entre masculino $(43,9 \%)$ e feminino $(56,1 \%)$.

Acerca da distribuição por ciclo de vida, houve uma concentração de casos relacionados ao idoso de 60 a 69 anos $(45,6 \%)$ e adulto de 20 a 59 anos (38,7\%). Em seguida, apareceu a adolescência (10 a 19 anos) e a infância (0 a 9 anos) em igual percentual (5,3\%). Esta distribuição não representa o contexto da população brasileira de acordo com o Censo Populacional de $2000^{19}$, onde as maiores concentrações aparecem de 20 a 59 anos $(51,3 \%)$ e de 10 a 19 anos $(20,8 \%)$, seguidas por crianças de 0 a 9 anos $(19,4 \%)$ e idosos $(8,6 \%)$.

A distribuição da ocupação dos personagens, realizada de acordo com o Critério Padrão de Classificação Econômica ${ }^{18}$, se encontra na Tabela 1.

TABELA 1

Distribuição dos personagens das estações de simulação da I turma do curso de Medicina da UFSCar, segundo ocupação, 2009.

\begin{tabular}{lcc}
\hline \multicolumn{1}{c}{ Ocupação dos personagens } & $\mathbf{n}$ & $\mathbf{\%}$ \\
\hline Aposentado & 18 & 31,6 \\
Empregado & 14 & 24,6 \\
Dona-de-casa & 08 & 14,0 \\
Estudante & 04 & 7,0 \\
Desempregado & 04 & 7,0 \\
NA (não se aplica) & 04 & 7,0 \\
Autônomo & 03 & 5,3 \\
Trabalhadores manuais sem capacitação & 01 & 1,7 \\
NC (não consta) & 01 & 1,7 \\
\hline Total & $\mathbf{5 7}$ & $\mathbf{1 0 0 , 0}$ \\
\hline
\end{tabular}

A maioria dos personagens era aposentada $(31,6 \%)$, seguida por aqueles que possuíam ocupação com registro em carteira de trabalho $(24,6 \%)$. Este percentual está aquém dos dados de estudos sobre o tipo de vínculo empregatício, que aponta $42,4 \%$ dos trabalhadores com carteira assinada em $2006^{20}$. Em seguida, 16,0\% eram estudantes. Também chama a atenção o pequeno percentual de desempregados, visto que este percentual em São Paulo é de 13,8\% $\%^{21}$. O elevado percentual de aposentados está condizente com a faixa etária predo- 
minantemente trabalhada nas ES, sendo que, em 2000, 66,8\% de brasileiros com 60 anos de idade ou mais se encontravam aposentados $^{22}$. A distorção da distribuição da ocupação dos personagens em relação à população brasileira pode ser explicada pelo predomínio de personagens com idade superior a 60 anos, diferentemente da realidade local, regional e nacional.

Com relação ao aspecto religioso, as ES permitiram aos estudantes a aproximação com diferentes religiões, com predomínio da católica $(47,4 \%)$. Não possuíam religião 38,6\% dos personagens das ES. Segundo dados do Censo $2000^{19}$, há predomínio da religião católica no Brasil, havendo coerência com as situações apresentadas.

Sobre a cor da pele, 68,4\% eram da cor branca, 10,5\% da cor parda, e 1,7\% da cor negra. Quanto aos desfechos, 68,4\% corresponderam a casos de pacientes em tratamento. Em 31,6\% das situações, a história não apresentava desfecho.

No que se refere ao processo saúde-doença, em 28,1\% das ES trabalhou-se o tratamento e na mesma porcentagem a promoção. Em $21,0 \%$ dos casos, apareceram tratamento e prevenção associados, em 12,3\%, prevenção, e em 7,0\%, associação entre prevenção, tratamento e reabilitação. O enfoque em tratamento continua alto, mas a promoção acompanha essa ocorrência, o que está de acordo com as propostas atuais de formação de profissionais de saúde, que preconizam a ampliação das dimensões do cuidado, principalmente na promoção à saúde e prevenção de doenças ${ }^{23,24}$. Vale ressaltar que, neste currículo, a atividade de Prática Profissional desde o primeiro ano do curso tem forte presença dos aspectos preventivos e de promoção à saúde ${ }^{14}$

Na categoria condição saúde e doença, $82,5 \%$ dos casos apresentavam algum tipo de patologia, enquanto em somente $17,5 \%$ foram trabalhadas condições de saúde. As condições foram codificadas a partir do CID e estão sistematizadas na Tabela 2.

O maior percentual se concentrou no Capítulo IV — Doenças endócrinas, nutricionais e metabólicas, alcançando 28,8\%, seguido do Capítulo IX - Doenças do aparelho circulatório $(13,5 \%)$. Esta distribuição está condizente com o Suplemento de Saúde da PNAD 2003, que estimou que 29,9\% dos brasileiros eram portadores de alguma doença crônica ${ }^{25}$. Embora as ES tenham possibilitado a abordagem da principal causa de óbitos registrados em 2008 no Brasil, que são as doenças do aparelho circulatório, responsáveis por 29,5\% das mortes, não trabalharam ou não enfatizaram numa proporção adequada as outras três principais condições relacionadas a óbitos: Capítulo II - Neoplasias (15,6\%), Capítulo XX - Causas externas de morbidade e de mortalidade (12,5\%) e Capítulo X - Doenças do aparelho respiratório $(9,8 \%)^{26}$.
TABELA 2

Distribuição das Condições de Saúde/Doença dos Personagens das Estações de Simulação da I Turma do Curso de Medicina da UFSCar, 2009

\begin{tabular}{llc}
\hline Capítulo & CID-10 & $\%$ \\
\hline $\begin{array}{l}\text { Capítulo I Algumas doenças } \\
\text { infecciosas e parasitárias (A00-B99) }\end{array}$ & $\begin{array}{l}\text { A00-A09 Doenças infecciosas } \\
\text { intestinais }\end{array}$ & 1,8 \\
$\begin{array}{l}\text { Capítulo IV Doenças endócrinas, } \\
\text { nutricionais e metabólicas }\end{array}$ & $\begin{array}{l}\text { E00-E07 Transtornos da glândula } \\
\text { tireóide }\end{array}$ & 0,9 \\
$\begin{array}{l}\text { Capítulo V Transtornos mentais e } \\
\text { comportamentais (F00-F99) }\end{array}$ & E10-E14 Diabetes mellitus & 9,9 \\
& & 9,9
\end{tabular}

E66-E68 Obesidade e outras formass de heperalimentação E70-E90 Distúrbios metabólicos $\quad 8,1$ F10-F19 Transtornos mentais e $\quad 0,9$ comportamentais devidos ao uso de substância psicoativa

Capítulo VIII Doenças do ouvido e H90-H95 Outros transtornos do da apófise mastóide (H60-H95) ouvido

Capítulo IX Doenças do aparelho I10-I15 Doenças hipertensivas circulatório (I00-I99)

Capítulo X Doenças do aparelho respiratório (J00-J99)

I30-I52 Outras formas de doença do coração

Capítulo XI Doenças do aparelho I60-I69 Doenças cerebrovasculares digestivo (K00-K93)

Capítulo XIII Doenças do sistema os teomuscular e do tecido conjuntivo (M00-M99)

Capítulo XIV Doenças do aparelho geniturinário (N00-N99)

Capítulo XVI Algumas afecções originadas no período perinatal (P00-P96)

Capítulo XVII Malformações congênitas, deformidades e anomalias cromossômicas (Q00-Q99)

J00-J06 Infecções agudas das vias aéreas superiores

K55-K63 Outras doenças dos intestinos

M20-M25 Outos transtornos articulares

M70-M79 Outros transtornos dos tecidos moles

N40-N51 Doenças dos órgãos genitais masculinos

N70-N77 Doenças inflamatórias dos 0,9 órgãos pélvicos femininos

P20-P29 Transtornos respiratórios e cardiovasculares específicos do período perinatal

Q20-Q28 Malformações congênitas do aparelho circulatório

R00-R09 Sintomas e sinais relativos ao aparelho circulatório e respiratório

R10-R19 Sintomas e sinais relativos ao aparelho digestivo e ao abdome

Capítulo XVIII Sintomas, sinais e achados anormais de exames clínicos e de laboratório, não classificados em outra parte (R00-R99) 


\begin{tabular}{llc}
\hline Capítulo & CID-10 & $\%$ \\
\hline $\begin{array}{l}\text { Capítulo XX Causas externas } \\
\text { de morbidade e de mortalidade } \\
\text { (V01-Y98) }\end{array}$ & R50-R69 Sintomas e sinais gerais & 10,8 \\
$\begin{array}{l}\text { Capítulo XXI Fatores que } \\
\text { influenciam o estado de saúde e o } \\
\text { contato com os serviços de saúde } \\
\text { (Z00-Z99) }\end{array}$ & $\begin{array}{l}\text { Z30-Z39 Pessoas em contato com os de saúde em circustâncias } \\
\text { relacionadas com a reprodução }\end{array}$ & 3,6 \\
& $\begin{array}{l}\text { Z55-Z65 Pessoas com riscos } \\
\text { potenciais à saúde relacionados }\end{array}$ & 9,1 \\
& $\begin{array}{l}\text { com circunstâncias socioeconômicas } \\
\text { e psicossociais }\end{array}$ & \\
& $\begin{array}{l}\text { Z70-Z76 Pessoas em contato com } \\
\text { os serviços de saúde em outras } \\
\text { circunstâncias }\end{array}$ & 0,9 \\
& & 1,8 \\
\hline Auto-medicação & & $\mathbf{1 0 0 , 0}$ \\
\hline TOTAL & &
\end{tabular}

Acerca do contexto onde os casos ocorreram, houve predominância da Atenção Primária à Saúde (93,0\%), demonstrando coerência com o cenário de inserção dos estudantes na atividade curricular de Prática Profissional do primeiro ao quarto ano, pois estavam desenvolvendo atividades em Unidade de Saúde da Família (USF) e Unidade Básica de Saúde (UBS). Nesta, os estudantes passaram em esquema de rodízio pelas áreas de Saúde da Mulher, Criança e Adulto e Idoso.

Todas as estações abordaram a dimensão biológica, sendo 19,4\% das situações com foco exclusivamente biológico. Em $49,1 \%$, esta apareceu associada às dimensões psicológica e social, em 17,5\% somente articulada à psicológica, e em 14,0\% juntamente à psicológica. Esses resultados são condizentes com as expectativas, considerando que o aspecto biológico deve permear a quase totalidade das ES e, quando possível, associado a situações psicológicas e socioculturais que possam interferir no processo saúde-doença.

Quanto às áreas de competência, de acordo com o projeto pedagógico, era previsto que os problemas abordassem tanto os Cuidados às Necessidades Individuais de Saúde, quanto os Cuidados às Necessidades Coletivas de Saúde, a Organização do Trabalho em Saúde, a Gerência do Cuidado e a Educação. Porém, os casos trabalhados focalizaram principalmente o Cuidado individual, que apareceu isoladamente em $49,2 \%$ das ES e em 36,8\% associado à Educação, e somente Educação em 14,0\% dos casos. As demais áreas não foram abordadas. Isto se explica pelo fato de a prática médica ter seu foco principal no cuidado individual e pela ESPP ser a atividade privilegiada para a formação clínica. Nesta análise, este foco apareceu em 86,0\% das ES.

Para a análise da variável área de conhecimento, foram consideradas as áreas de conhecimento descritas no projeto pedagógico do curso de Medicina da UFSCar, que são as seguintes: anatomia; anatomia patológica; biologia molecular; bioquímica; embriologia humana; ética e bioética; farmacologia terapêutica; fisiologia e biofísica; educação e pesquisa em saúde; gestão em saúde; genética médica; histologia; imunologia; informática em saúde; inglês instrumental em saúde; medicina legal; microbiologia; parasitologia; semiologia clínica. Nos casos em que não ficaram claras as áreas de conhecimento, os conteúdos foram classificados como indefinidos. Quando estava evidente a área de conhecimento, mas esta não fazia parte das áreas elencadas no PPP, foram classificadas como outras (Quadro 1).

\section{QuAdro 1}

Áreas de conhecimento abordadas nas estações de simulação, segunda série, da I Turma do Curso de Medicina da UFSCar, 2009

\begin{tabular}{|l|c|c|c|c|}
\hline \multicolumn{1}{|c|}{ Áreas de conhecimento } & 10 Ano & 20 Ano & 3ㅜ Ano & 4. \\
\hline Anatomia \\
\hline Anatomomia patológica & $\mathrm{N}$ & $\mathrm{N}$ & $\mathrm{N}$ & $\mathrm{S}$ \\
\hline Biologia molecular & $\mathrm{N}$ & $\mathrm{N}$ & $\mathrm{N}$ & $\mathrm{S}$ \\
\hline Bioquímica & $\mathrm{N}$ & $\mathrm{N}$ & $\mathrm{N}$ & $\mathrm{N}$ \\
\hline Embriologia humana & $\mathrm{N}$ & $\mathrm{N}$ & $\mathrm{N}$ & $\mathrm{N}$ \\
\hline Ética e bioética & $\mathrm{N}$ & $\mathrm{N}$ & $\mathrm{N}$ & $\mathrm{N}$ \\
\hline Farmacologia terapêutica & $\mathrm{N}$ & $\mathrm{S}$ & $\mathrm{N}$ & $\mathrm{S}$ \\
\hline Fisiologia e Biofísica & $\mathrm{S}$ & $\mathrm{N}$ & $\mathrm{N}$ & $\mathrm{S}$ \\
\hline Educação e pesquisa em saúde & $\mathrm{N}$ & $\mathrm{N}$ & $\mathrm{N}$ & $\mathrm{S}$ \\
\hline Gestão em saúde & $\mathrm{N}$ & $\mathrm{S}$ & $\mathrm{S}$ & $\mathrm{N}$ \\
\hline Genética médica & $\mathrm{S}$ & $\mathrm{N}$ & $\mathrm{N}$ & $\mathrm{S}$ \\
\hline Histologia & $\mathrm{N}$ & $\mathrm{N}$ & $\mathrm{N}$ & $\mathrm{N}$ \\
\hline Imunologia & $\mathrm{N}$ & $\mathrm{N}$ & $\mathrm{N}$ & $\mathrm{N}$ \\
\hline Informática em saúde & $\mathrm{N}$ & $\mathrm{N}$ & $\mathrm{N}$ & $\mathrm{N}$ \\
\hline Inglês instrumental em saúde & $\mathrm{N}$ & $\mathrm{N}$ & $\mathrm{N}$ & $\mathrm{N}$ \\
\hline Medicina Legal & $\mathrm{N}$ & $\mathrm{N}$ & $\mathrm{N}$ & $\mathrm{N}$ \\
\hline Microbiologia & $\mathrm{N}$ & $\mathrm{N}$ & $\mathrm{N}$ & $\mathrm{N}$ \\
\hline Parasitologia & $\mathrm{N}$ & $\mathrm{N}$ & $\mathrm{N}$ & $\mathrm{N}$ \\
\hline Semiologia Clínica & $\mathrm{N}$ & $\mathrm{N}$ & $\mathrm{N}$ & $\mathrm{N}$ \\
\hline Outras áreas de conhecimento & $\mathrm{S}$ & $\mathrm{S}$ & $\mathrm{S}$ & $\mathrm{S}$ \\
\hline $\begin{array}{l}\text { Indefinido (não está clara a área de } \\
\text { conhecimento e nem o conteúdo }\end{array}$ & $\mathrm{S}$ & $\mathrm{N}$ & $\mathrm{S}$ & $\mathrm{S}$ \\
\hline
\end{tabular}

N: não; S: sim

Verifica-se que em todos os anos ocorrem estações que permitem a abordagem da semiologia clínica, porém diversas áreas não são contempladas e outras em apenas alguns anos (anatomia, anatomia patológica, ética e bioética, farmacologia terapêutica, fisiologia e biofísica, educação e pesquisa em saúde, gestão em saúde).

Considerando que as ES têm como principal objetivo o desenvolvimento da área de competência do cuidado à saúde individual, com foco na construção do raciocínio clínico e nas tarefas de construção de história clínica, exame físico, levantamento de necessidades de saúde e plano de cuidados, e, ainda, que estes estão intimamente relacionados à área de conhecimento da semiologia clínica, é desejável que as estações em todos os anos possibilitem a abordagem de conteúdos da semiologia, tal como vem ocorrendo.

Desta forma, a não abordagem de algumas áreas de conhecimento é justificada, levando em conta que a ESPP não 
é a única estratégia de aprendizagem, ocorrendo de forma concomitante e complementar às atividades curriculares de situações-problema e prática profissional, que podem e devem abordar as áreas aqui não contempladas.

A ausência de conteúdos sobre ética nas ES do primeiro e do terceiro ano preocupa, pois a abordagem ética nas relações médico-paciente é uma constante que as ES poderiam contemplar em todos os anos, especialmente em um currículo que privilegia a prática profissional no mundo do trabalho real desde o início do curso.

\section{CONSIDERAÇÕES FINAIS}

A atividade de simulação da prática que utiliza atores na condição de pacientes simulados, desenvolvida em ambiente controlado e protegido, tem se mostrado uma estratégia pedagógica importante para a formação médica, uma vez que dá mais segurança ao estudante no desenvolvimento de suas habilidades, proporciona melhor feedback do professor ao estudante e protege o paciente, que, em muitas ocasiões, é usado simplesmente como objeto de aprendizagem.

A análise dos dados apontou predomínio de estações na área de conhecimento de Saúde do Adulto e Idoso e Saúde da Família e Comunidade. Esse resultado é esperado, pois o foco desta atividade é o desenvolvimento de habilidades clínicas. Por outro lado, houve fragilidade na abordagem das demais áreas. Quanto ao cenário das ES, áreas de competência e distribuição pelas dimensões biológica, psicológica e social, houve coerência com os objetivos da atividade curricular. Embora o enfoque do processo saúde-doença tenha sido o tratamento, as estações também trouxeram elementos de promoção e prevenção, em consonância com o movimento de ampliação das dimensões do cuidado. A distribuição das condições de saúde e doença nas ES, de acordo com o CID-10, é condizente com o perfil epidemiológico brasileiro ${ }^{27}$.

Nesta análise foi identificada a necessidade de uma readequação das ES do curso médico da UFSCar, elaboradas entre 2006 e 2009, no que diz respeito a áreas de conhecimento, faixa etária, ocupação, fases do processo saúde-doença e desfecho das situações. Também é preciso readequar as áreas de conhecimento do projeto pedagógico, pois vários conteúdos identificados pertenciam a áreas de conhecimento não existentes no projeto e que são importantes para a formação médica. Além disso, verificou-se a necessidade de caracterizar melhor as ementas das Estações de Simulação das áreas de conhecimento específicas, pois algumas vezes estas não estavam claras.

As ES devem ser bem estruturadas e representar a realidade da prática médica. Desta forma, esta investigação, ao analisar as ES trabalhadas no curso médico da UFSCar, contribuiu com a formulação de variáveis e critérios que devem orientar a construção das ES, para que estas representem o melhor possível a realidade da prática profissional.

\section{REFERÊNCIAS}

1. Carraccio C, Wolfsthal SD, Englander R, Ferentz K, Martin C. Shifting paradigms: from Flexner to competencies. Acad Med.2002;77(5):361-7.

2. Ali J, Al Ahmadi K, Williams JI, Cherry RA. The standardized live patient and mechanical patient models-their roles in trauma teaching. J Trauma. 2009;66(1):98-102.

3. Rees C, Sheard C, Mcpherson A. Medical students' views and experiences of methods of teaching and learning communication skills. Patient Educ Couns. 2004;54(1)119-21.

4. Varga CRR, Almeida VC, Germano CMR, Melo DG, Chachá SGF, Souto BGA, Fontanella BJB, Lima VV. Relato de experiência: o uso de simulações no processo de ensino-aprendizagem em medicina. Rev Bras Educ Med. 2009;33(2)291-7.

5. Unalan PC, Uzuner A, Cifcili S, Akman M, Hancioglu S, Thulesius HO. Using theatre in education in a traditional lecture oriented medical curriculum. BMC Med Educ [periódico na internet]. 2009 [acesso em 16 jun 2010];9(73):[cerca de 5 páginas]. Disponível em: http://www.ncbi.nlm.nih. gov/pmc/articles/PMC2803161

6. Jacobsen T, Baerheim A, Lepp MR, Schei E. Analysis of role-play in medical communication training using a theatrical device the fourth wall. BMC Med Educ [periódico na internet]. 2006 [acesso em 16 jun. 2010];6(51):[cerca de 8 páginas].Disponível em: http:/ / www.ncbi.nlm.nih.gov / pmc/articles/PMC1621062/

7. Botezatu M, Hult H, Tessma MK, Fors UG. Virtual patient simulation for learning and assessment: Superior results in comparison with regular course exams. Med Teach [periódico na internet]. 2010. [acesso em 28 mar. 2011];32(10):84550. Disponível em: http://www.ncbi.nlm.nih.gov/pubmed/20854161

8. Wayne DB, Butter J, Siddall VJ, Fudala MJ, Wade LD, Feinglass J, McGaghie WC. Mastery learning of advanced cardiac life support skills by internal medicine residents using simulation technology and deliberate practice. J Gen Intern Med. [periódico na internet]. 2006 [acesso em 16 jun. 2010];21(3): 251-6. Disponível em: http:/ /www.ncbi.nlm. nih.gov/pubmed/16637824

9. Unroe MA, Shofer SL, Wahidi MM. Training for endobronchial ultrasound: methods for proper training in new bronchoscopic techniques. Curr Opin Pulm Med [periódico na internet]. 2010 [acesso em 16 jun. 2010];16(4): 295-300. Disponível em: http:/ / www.ncbi.nlm.nih.gov/pubmed/20531196

10. Curran VR, Butler R, Duke P, Eaton WH, Moffatt SM, Sherman GP, Pottle M. Evaluation of the usefulness of simu- 
lated clinical examination in family-medicine residency program. Med Teach. 2007;29(4)406-7.

11. Dornan T, Littlewood S, Margolis SA, Scherpbier A, Spencer J, Ypinazar V. How can experience in clinical and community settings contribute to early medical education? A BEME Systematic review. Med Teach. 2006;28(1):3-18.

12. Kurtz S. Teaching and learning communication in veterinary medicine. J Vet Med Educ. 2006;33(1)11-9.

13. Troncon LEA, Maffei CML. A incorporação de recursos de simulação no curso de graduação em medicina da Faculdade de Medicina de Ribeirão Preto - USP. Medicina (Ribeiräo Preto). 2007;40(2): 153-61.

14. Universidade Federal de São Carlos. Coordenação do Curso de Medicina. Caderno do Curso de Medicina. São Carlos: USFCar; 2006.

15. Howley LD, Martindale J. The efficacy of standardized patient feedback in clinical teaching. A mixed methods analysis. Med Educ Online [periódico na internet]. 2004 [acesso em 16 jun. 2010];9(18):[cerca de 1 tela]. Disponível em: http:/ / www.med-ed-online.org.

16. Smith SR Cookson J, Mckendree J, Harden RM. Patient-centred learning-back to the future. Med Teach. 2007;29(1)33-7.

17. Hoffmann J. Avaliar para promover: as setas do caminho. Porto Alegre: Mediação; 2001.

18. Associação Brasileira de Empresas de Pesquisa. Critério padrão de classificação econômica Brasil, 2008 [on line]. [S.l:s.n]; 2007 [acesso em 16 jun. 2010]. Disponível em: http:/ /www. viverbem.fmb.unesp.br/docs/classificacaobrasil.pdf

19. Instituto Brasileiro de Geografia e Estatística. Censos demográficos 2000 [homepage]. Brasília:IBGE;[acesso em 16 jun. 2010]. Pirâmide Etária; [cerca de 1 tela]. Disponível em: http://www.ibge.gov.br/brasil_em_sintese/populacao02.htm.

20. Hallak J, Namir K, Kozovits L. Setor e emprego informal no Brasil: Análise dos resultados da nova série do sistema de contas nacionais (2000-2006). [on line]. [S.1:s.n];[acesso em 16 jun. 2010]. 2006 [cerca de 23 páginas]. Disponível em: http://www.ie.ufrj.br/datacenterie/pdfs/seminarios/pesquisa/texto1310.pdf

21. Departamento Intersindical de Estatística e Estudos Sócio-Econômicos. Taxa de desemprego total, por sexo. [on line]. [S.l:s.n];[acesso em 16 jun. 2010]. 2009 [cerca de 1 tela]. Disponível em: http://turandot.dieese.org.br/icv/ TabelaPed?tabela $=5$

22. Instituto Brasileiro de Geografia e Estatística [homepage]. Brasília: IBGE; [acesso em 16 jun. 2010]. Sala de imprensa: Indicadores Sociais Municipais - uma análise dos resultados da amostra do Censo. Indicadores Sociais Municipais mostram importância das aposentadorias no rendimento familiar mensal nas áreas rurais; [cerca de 1 tela]. Disponível em: http://www.apm.org.br/aberto/educacaomedica_interna.aspx?id=121

23. Brasil. Ministério da Educação. Conselho Nacional de Educação. Câmara de Educação Superior. Resolução CNE/ CES no 4/2001. Diretrizes Curriculares Nacionais do Curso de Graduação em Medicina. Diário Oficial da União, Poder Executivo, Brasília, DF, 9 nov. 2001. Seção 1, p. 38.

24. Brasil. Ministério da Saúde. Ministério da Educação. Pró-saúde: programa nacional de reorientação da formação profissional em saúde. Brasília. [on line]. 2005. [acesso em 28 mar. 2011]. Disponível em: http:/ /www.prosaude.org/ rel/pro_saude1.pdf

25. Instituto Brasileiro de Geografia e Estatística [homepage]. Brasília:IBGE;[acesso em 16 jun. 2010]. 2005. Comunicação Social. Acesso e utilização de serviços de saúde - 2003. Doenças crônicas atingem quase um terço da população brasileira [cerca de 1 tela]. Disponível em: http://www. ibge.gov.br/home/presidencia/noticias/noticia_impressao.php?id_noticia $=370$

26. Ministério da Saúde. Informações de Saúde [homepage]. Brasília: MS; [acesso em 19 jun. 2010]. DATASUS. Mortalidade: Dados preliminares Brasil. Óbitos por ocorrência por capítulo CID-10 segundo região, 2008. [cerca de 1 tela]. Disponível em: http:/ / tabnet.datasus.gov.br/cgi/sim/pobt10descr.htm

27. Organização Mundial da Saúde. Classificação Estatística Internacional de Doenças e Problemas Relacionados à Saúde. Décima Revisão. Versão 2008. Volume I. [on line]. 2008 [acesso em 25 abr. 2011]. Disponível em: http://www.datasus.gov.br/cid10/v2008/cid10.htm

\section{CONTRIBUIÇÃO DOS AUTORES}

As autoras trabalharam juntas nas diferentes etapas de produção do manuscrito. A autora principal fez a articulação das partes produzidas e a revisão final do artigo.

\section{CONFLITO DE INTERESSES}

Declarou não haver.

\section{ENDEREÇO PARA CORRESPONDÊNCIA}

Roseli Ferreira da Silva

Departamento de Medicina — Universidade Federal de São Carlos

Rod. Washington Luís, km 235 - CP 676 - São Carlos

CEP 13565-905 — SP

E-mail: roselifs@ufscar.br 